\title{
Synthesis of novel phase transfer catalysts derived from proline-mandelic acid/tartaric acid: their evaluation in enantioselective epoxidation and Darzen condensation
}

\author{
DEEPAK P MAHAJAN ${ }^{\mathrm{a}, *}$, HIMANSHU M GODBOLE ${ }^{\mathrm{a}}$, GIRIJ P SINGH ${ }^{\mathrm{a}}$ and \\ GAUTHAM G SHENOY \\ ${ }^{a}$ Lupin Limited, 46 \& 47A, Village Nande, Taluka Mulshi, Pune, Maharashtra 412 115, India \\ ${ }^{b}$ Manipal Academy of Higher Education, Manipal, Karnataka 576 104, India \\ E-mail: deepakmahajan@lupin.com; dmahajan007@gmail.com
}

MS received 6 November 2018; revised 24 December 2018; accepted 29 January 2019; published online 23 February 2019

\begin{abstract}
Herein, we have described the synthesis of novel chiral cyclic phase transfer catalysts (PTCs). These catalysts are synthesized from proline, mandelic acid and tartaric acid by using simple synthetic methods with competitive yields. These chiral cyclic phase transfer catalysts have been characterized by ${ }^{1} \mathrm{H}$ NMR and ${ }^{13} \mathrm{C}$ NMR spectroscopy, IR spectroscopy and elemental analysis. The effectiveness of these novel chiral cyclic phase transfer catalysts was evaluated by applying them in the enantioselective epoxide synthesis from $\alpha$, $\beta$ unsaturated ketone (chalcone) and Darzens condensation. The obtained results show that the synthesized derivatives of proline, mandelic acid and tartaric acid are effectual as PTCs.
\end{abstract}

Keywords. Proline; mandelic acid; tartaric acid; chiral phase transfer catalysts; enantioselective epoxidation; Darzen condensation.

\section{Introduction}

Phase Transfer Catalysis (PTC) methodology furnishes many captivating advantages in synthetic chemistry. ${ }^{1,2}$ This methodology contributes to 'Green chemistry' - as it involves organic solvent-free processes and avoids the use of hazardous compounds. ${ }^{3}$ Progressively, the importance of chiral PTC is increasing in asymmetric chemical synthesis. Several chiral PTCs are reported in the literature, like cinchona alkaloid derivatives, binaphthyl derivatives, tartaric acid derivatives, chiral crown ethers and some spiro type ammonium compounds. ${ }^{4}$ Chiral PTCs are employed in various asymmetric syntheses like enantioselective alkylation, epoxidation, aldol condensation and Darzens condensation. ${ }^{4,5}$

The structural designing of all existing chiral PTCs reported in the literature is based on the geometry of quaternary nitrogen ${ }^{5}$ because it has the same geometry as the tetrahedral carbon. If the nitrogen of a quaternary ammonium salt is considered as the center of a tetrahedron, the phase transfer catalyst should be structured in a such way that it should provide steric hindrance which arrests the close approach of the counter ion to the three faces of this tetrahedral quaternary nitrogen, while the remaining fourth face should be sufficiently free to make close contact between the quaternary nitrogen and the counter ion. It would be an advantage for getting the enantioselectivity, if there are binding surfaces (hydroxy or alkoxy groups) available nearby to the quaternary nitrogen for attractive van der Waals interaction.

With reference to this, we have designed and synthesized novel chiral PTCs from proline, mandelic acid and tartaric acid (Figure 1, 2 and 3). These chiral phase transfer catalysts are designed and synthesized in such a way that out of four sides, three sides of quaternary nitrogen are blocked with cyclic groups and one aromatic group at $\alpha$ or $\beta$ position to the nitrogen, whereas only one side is available to form ion pairing with an anion. These PTCs have $-\mathrm{OH}$ or -OR group at the $\beta$ position to the quaternary nitrogen which can help to form hydrogen bonding or attractive van der Waals interaction to get an advantage for enhancing the enantioselectivity.

\footnotetext{
*For correspondence

Electronic supplementary material: The online version of this article (https://doi.org/10.1007/s12039-019-1597-6) contains supplementary material, which is available to authorized users.
} 


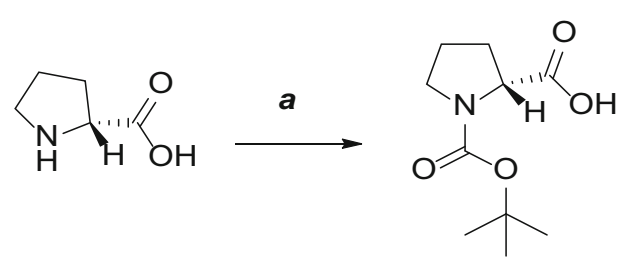

D-proline
(1)

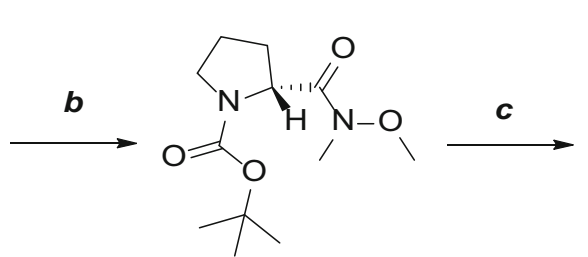

(1a)

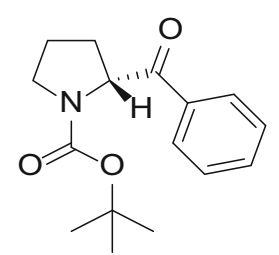

(1b)

(1d)

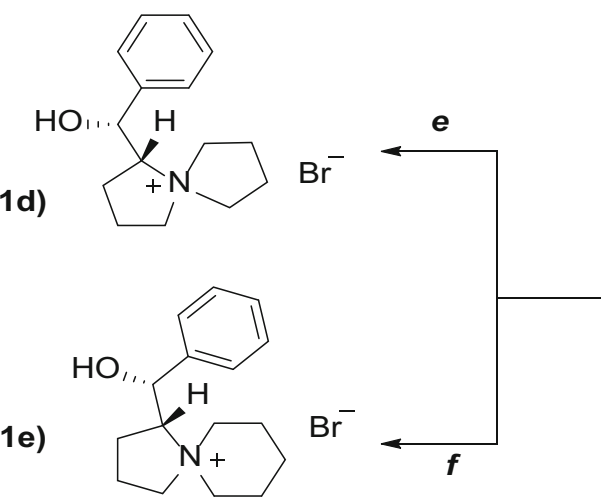

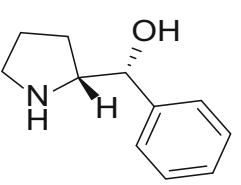

(1c)

Figure 1. Synthesis of chiral phase transfer catalysts from Proline (1d and 1e).

\subsection{Synthesis of novel chiral PTCs from proline}

The synthesis of novel chiral PTCs from proline involved multi-step synthesis (Figure 1). First the amino group of Proline was protected by $\mathrm{BoC}$ to get (tert-butoxycarbonyl)-D-proline (1) which was further converted to Weinreb's amide (1a). Weinreb's amide (1a) was then converted to Ketone (1b) by using Grignard reagent. The amino group of Ketone (1b) was first deprotected and then reduced to get the racemic alcohol which was purified by column chromatography to get the desired diasteriomer of alcohol (1c). The chiral alcohol (1c) was treated with respective di halo compound to get cyclized quaternary salts. The crude cyclized quaternary salts were purified by recrystallization to get pure chiral cyclized quaternary ammonium salts $(\mathbf{1 d})$ and (1e).

Reagents and conditions: $\boldsymbol{a}-\mathrm{DCM},(\mathrm{BoC})_{2} \mathrm{O}(1.1$ mole. eq), $\mathrm{Et}_{3} \mathrm{~N}$ (1.1 mole. $\left.\mathrm{Eq}\right), 25-30^{\circ} \mathrm{C}, 6$ h. $\boldsymbol{b}-$ DCM, CDI (1.2 mole. eq), $\mathrm{CH}_{3} \mathrm{NOCH}_{3}$. $\mathrm{HCl}$ (1.2 mole. eq), $25-30^{\circ} \mathrm{C}, 3 \mathrm{~h} . \boldsymbol{c}-\mathrm{THF}, 1.0 \mathrm{M} \mathrm{PhMgBr}$ in THF (2.0 mole. eq), -45 to $-55^{\circ} \mathrm{C}, 1.5$ h. $\boldsymbol{d}-\mathrm{EtOAc}, \mathrm{HCl}$ (3.0 mole. eq), $\mathrm{MeOH}, \mathrm{NaBH}_{4}\left(2.0\right.$ mole. eq), $0-20^{\circ} \mathrm{C}$ 1.5 h. $\boldsymbol{e}-\mathrm{K}_{2} \mathrm{CO}_{3}$ (2.0 mole. eq), 1,4 $\mathrm{Br}\left(\mathrm{CH}_{2}\right)_{4} \mathrm{Br}(1.1$ mole. eq), ACN, Reflux, 12 h. $f-\mathrm{K}_{2} \mathrm{CO}_{3}$ (2.0 mole. eq), $\operatorname{Br}\left(\mathrm{CH}_{2}\right)_{5} \mathrm{Br}$ (1.1 mole. eq), ACN, Reflux, $12 \mathrm{~h}$.

\subsection{Synthesis of novel chiral PTCs from mandelic acid}

The synthesis of chiral PTCs from Mandelic acid involved multi-step synthesis (Figure 2). The Mandelic acid was converted to Ketone (2) via Weinreb's amide formation. Reductive amination of Ketone (2) was done to get racemic amine which was purified by column chromatography to get the chiraly pure Amine (2a). The pure Amine (2a) was treated with respective di halo compounds to get cyclized quaternary salts ( $\mathbf{2} \mathbf{b}$ and $\mathbf{2 c}$ ). The crude cyclized quaternary salts were purified by recrystallization to get pure chiral cyclized quaternary ammonium salts (2b) and (2c).

Reagents and conditions: $\boldsymbol{g}$ - CDI (1.2 mole. eq), $\mathrm{CH}_{3} \mathrm{NOCH}_{3}$. $\mathrm{HCl}$ (1.2 mole. eq), DCM, $25-30^{\circ} \mathrm{C}, 2 \mathrm{~h}$. $\boldsymbol{h}-1.0 \mathrm{M} \mathrm{PhMgBr}$ in THF (2.5 mole. eq), THF, -55 to $-65^{\circ} \mathrm{C}, 2$ h. $\boldsymbol{i}-2.0 \mathrm{M} \mathrm{MeNH}_{2}$ in $\mathrm{MeOH}$ (8.54 mole. eq), $6 \mathrm{~h}, \mathrm{NaBH}_{4}\left(2.0\right.$ mole. eq), $2 \mathrm{~h}, 0-10^{\circ} \mathrm{C} . \boldsymbol{j}-\mathrm{K}_{2} \mathrm{CO} 3$ (2.0 mole. eq), $\mathrm{Br}\left(\mathrm{CH}_{2}\right)_{4} \mathrm{Br}$ (1.1 mole. eq), $\mathrm{ACN}$, Reflux, 12 h. $\boldsymbol{k}-\mathrm{K}_{2} \mathrm{CO}_{3}$ (2.0 mole. eq), $\mathrm{Br}\left(\mathrm{CH}_{2}\right)_{6} \mathrm{Br}$ (1.1 mole. eq), ACN, Reflux, $12 \mathrm{~h}$.

\subsection{Synthesis of novel chiral PTCs from tartaric acid}

The synthesis of chiral PTCs from tartaric acid via multi-step synthesis is shown in Figure 3. The protected Diethyl tartrate compound (3) was treated with Pyrrolidine and Piperazine to get Amide compounds (3a) and $(\mathbf{3 b})$ respectively. The Amides (3a) and (3b) were reduced to get respective secondary Amines (3c) and $(\mathbf{3 d})$. The secondary amines $(\mathbf{3 c})$ and $(\mathbf{3 d})$ were reacted with benzyl bromide to get crude cyclized quaternary salts. The crude cyclized quaternary salts were purified by recrystallization to get pure chiral cyclized quaternary ammonium salts from tartaric acid (3e) and (3f). 
<smiles>CC(C)C</smiles>

(R)-mandelic acid<smiles>O=C(c1ccccc1)[C@H](O)c1ccccc1</smiles>

(2)<smiles>CNC(c1ccccc1)[C@@H](O)c1ccccc1</smiles>

(2a)

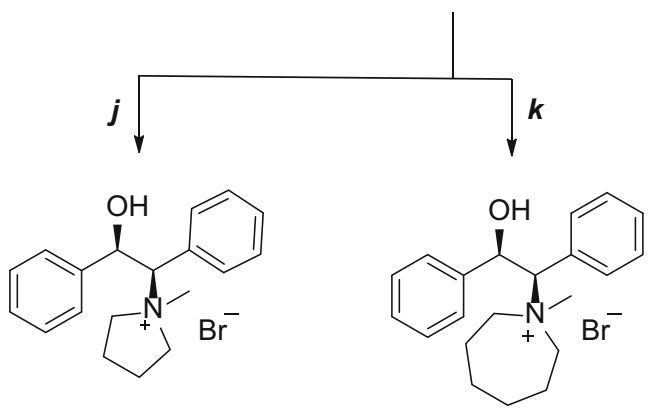

(2b)

Figure 2. Synthesis of chiral phase transfer catalysts from Mandelic acid (2b and 2c).

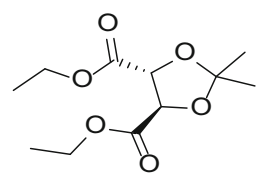

(3) Protected diethyl tartrate

(3a)<smiles>C1CCNCC1</smiles><smiles>CCCNC(=O)[C@@H]1OC(C)(C)O[C@@H]1C(=O)N1CCCC1</smiles>

(3c)<smiles>CC1(C)O[C@@H](CN2CCCC2)[C@H](CN2CCCC2)O1</smiles>

$$
p
$$<smiles>CC1(C)OC(CN2CCCC2)C(CN2CCCC2Br)O1</smiles>

(3e)

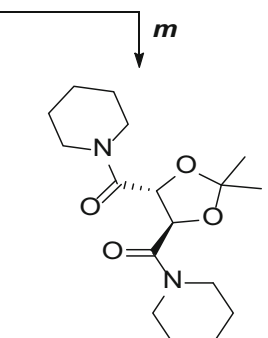

(3b)

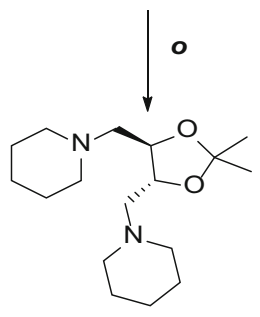

(3d)
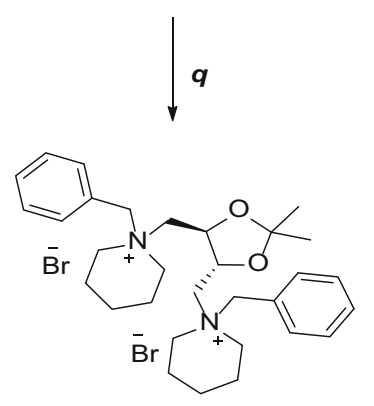

(3f)

Figure 3. Synthesis of chiral phase transfer catalysts from tartaric acid (3e and $\mathbf{3 f})$. 


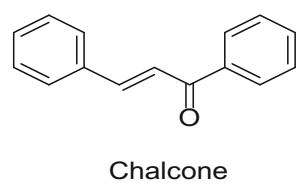

Chalcone

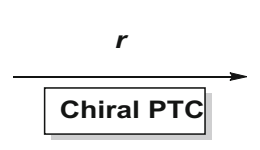

Chiral PTC

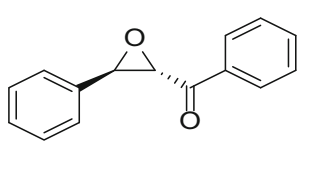

(4)

ee $73-87 \%$, yield $80-95 \%$<smiles>O=Cc1ccccc1</smiles>

Benzaldehyde<smiles>O=C(CCl)c1ccccc1</smiles>

2-chloro-1-phenylethan-1-one

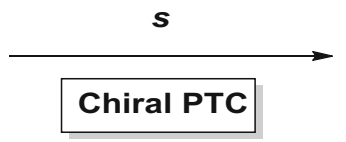

Chiral PTC

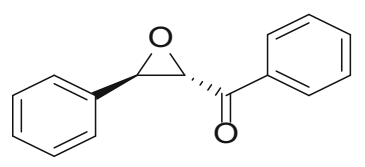

(4)

ee $72-84 \%$, yield $80-92 \%$

Figure 5. Darzen condensation to get chiral epoxide (4).

Reagents and conditions: $\boldsymbol{l}$ - Pyrrolidine $(6.0$ mole. eq), $20-30^{\circ} \mathrm{C}, 10 \mathrm{~h} . \boldsymbol{m}$ - Piperazine (6.0 mole. eq), 20-30 ${ }^{\circ} \mathrm{C}$, 10 h. $n$-LAH (3.0 mole. eq), Dioxan, Reflux, 12 h. $\boldsymbol{o}$ - LAH (3.0 mole. eq), Dioxan, Reflux, 12 h. $\boldsymbol{p}$ $-\mathrm{K}_{2} \mathrm{CO}_{3}$ (3.0 mole. eq), $\mathrm{PhCH}_{2} \mathrm{Br}$ (2.2 mole. eq), $\mathrm{ACN}$, Reflux, 10h. $\boldsymbol{q}-\mathrm{K}_{2} \mathrm{CO}_{3}$ (3.0 mole. eq), $\mathrm{PhCH}_{2} \mathrm{Br}(2.2$ mole. eq), ACN, Reflux, $10 \mathrm{~h}$.

\subsection{Synthesis of chiral epoxide by using novel chiral PTCs}

Enantioselective epoxidation of $\alpha, \beta$ unsaturated chalcone and chiral epoxide synthesis by Darzens reaction by using PTCs having chiral quaternary ammonium salts have already been reported in the literature. ${ }^{6}$ So, to check the effectiveness of these novel chiral PTCs, these catalysts have been applied to the enantioselective epoxidation of $\alpha, \beta$ - unsaturated chalcone (Figure 4) and for Darzen condensation (Figure 5), in which enantioselective epoxide (4) was synthesized.

1.4a Synthesis of chiral epoxide (4) by epoxidation of $\alpha, \beta$-unsaturated ketone (chalcone): In chiral epoxide (4) synthesis, $\alpha, \beta$-Unsaturated ketone (Chalcone) was treated with an oxidant in the presence of novel PTC, using mild conditions (Figure 4).

Reagents and conditions: $r-10 \%$ aqs. $\mathrm{NaoCl}(2.0$ mole. eq), Toluene, $20-30{ }^{\circ} \mathrm{C}, 6-12 \mathrm{~h}$.

1.4b Synthesis of chiral epoxide (4) by Darzen condensation: The chiral epoxide (4) was synthesized by Darzen condensation, which involved condensation Benzaldehyde with 2-Chloro-1-phenylethan-1-one ( $\alpha$ halo ketone) in the presence of chiral PTC, using mild conditions (Figure 5).
Reagents and conditions: $s-\mathrm{LiOH}_{\mathrm{H}} \mathrm{O}$ (2.0 mole. eq), MTBE, 20-30 ${ }^{\circ} \mathrm{C}, 9-18 \mathrm{~h}$.

\section{Experimental}

\subsection{Materials and physical measurements}

(D)-proline, (R)-mandelic acid and protected diethyl tartrate were purchased from Sigma Aldrich. 1,4-dibromo butane, 1,5 dibromo pentane, 1,6-dibomo hexane, chalone (1,3diphenyl-propen-3-one), benzaldehyde, phenacyl chloride, di-tert-butyldicarbonate and triethyl amine were purchased from Avra synthesis (India). These chemicals were used directly without additional treatment. Commercial grade solvents were used for reaction and purification. ${ }^{1} \mathrm{H}$ NMR $(400 \mathrm{MHz}$ and $500 \mathrm{MHz})$ and ${ }^{13} \mathrm{C}$ NMR $(125 \mathrm{MHz})$ were obtained as solutions in deuterium substituted reagent on Bruker AVANCE III HD, Software- Topspin 3.5. Chemical shifts were reported in parts per million $(\mathrm{ppm}, \delta$ ). Melting points were recorded on Mettler Toledo MP-03 melting point apparatus having higher calibrated melting limit of $238^{\circ} \mathrm{C}$. IR spectra (FTIR) were recorded on a Perkin spectrum 400 FTIR spectrometer using liquid film $/ \mathrm{KBr}$ pellet or ATR method. The $\mathrm{C}, \mathrm{H}$ and $\mathrm{N}$ elemental analyses were performed on a Yanaco CHN FOER MT-3 element analyzer. The HPLC analysis were carried out on SHIMADZU-6AD by using Phenomenex chiral pack column and HPLC grade solvents (Isopropyl alcohol and n-Hexane) .

\subsection{Synthesis of chiral PTCs from proline}

2.2a Synthesis of (tert-butoxycarbonyl)-D-proline (1): To a slurry of D-proline $(25 \mathrm{~g}, 217 \mathrm{mmol}, 1.0 \mathrm{eq})$ in dichloromethane $(250 \mathrm{~mL})$, Di-tert-butyldicarbonate $(52.2 \mathrm{~g}$, $239 \mathrm{mmol}, 1.1 \mathrm{eq})$ followed by TEA $(33 \mathrm{~mL}, 239 \mathrm{mmol}$, $1.1 \mathrm{eq})$ were added at $25-30^{\circ} \mathrm{C}$ and stirred for $4 \mathrm{~h}$. After 
completion of reaction, $5 \%$ aqs citric acid solution $(300 \mathrm{~mL})$ was added and stirred. The layers were separated, the organic layer was dried over sodium sulphate and solvent removed in vacuum to obtain (tert-butoxycarbonyl)-D-proline (1) as a white solid $(45.4 \mathrm{~g}, 97 \%)$.

(1) - White solid, Yield 45.4 g, 97\%, M. p.: $129.7-131.4{ }^{\circ} \mathrm{C}$ (Lit. $\left.130-132^{\circ} \mathrm{C}\right)^{7} ;[\alpha]_{\mathrm{D}}=+72.5^{\circ}$ (c 1.00, acetic acid), (Lit. $[\alpha]_{\mathrm{D}}=-68^{\circ}(c 1.00 \text {, acetic acid for }(S) \text {-isomer })^{8} ;{ }^{1} \mathrm{H}$ NMR $\left(400 \mathrm{MHz}, \mathrm{CDCl}_{3}\right) \delta: 1.42(\mathrm{~s}, 4.5 \mathrm{H}), 1.48(\mathrm{~s}, 4.5 \mathrm{H})$, 1.89-1.99 (m, 2H), 2.01-2.14 (m, 1H), 2.25-2.31 (m, 1H), 3.36-3.57 (m, 2H), $4.23(\mathrm{dd}, J=8 \mathrm{~Hz}, 1 \mathrm{H}$, mixture of rotamers), 9.07 (bs, $1 \mathrm{H}$, exchangeable with $\left.\mathrm{D}_{2} \mathrm{O}\right) ;{ }^{13} \mathrm{C} \mathrm{NMR}$ $\left(125 \mathrm{MHz}, \mathrm{CDCl}_{3}\right): 174.7,153.5,79.1,58.8,46.5,30.7$ 28.5, 28.3, 24.3. IR (ATR) $\nu / \mathrm{cm}^{-1}, 3071,1718,1630,1419,1361$, 1124; Anal. calcu lated for $\mathrm{C}_{10} \mathrm{H}_{17} \mathrm{NO}_{4}$ : C, 55.80; H, 7.96; N, 6.51. Found: C, 55.76; H, 7.98; N, 6.50.

2.2b Synthesis of tert-butyl (R)-2-(methoxy(methyl) carbamoyl) pyrrolidine-1-carboxylate (1a): To a solution of protected Proline (1) $(25 \mathrm{~g}, 116 \mathrm{mmol}, 1.0 \mathrm{eq})$ in dichloromethane $(200 \mathrm{~mL})$, charged 1,1'-Carbonyl diimidazole $(22.61 \mathrm{~g}, 139 \mathrm{mmol}, 1.2 \mathrm{eq})$ and stirred for $1 \mathrm{~h} . \mathrm{N}$, Odimethyl hydroxylamine hydrochloride $(13.61 \mathrm{~g}, 139 \mathrm{mmol}$, 1.2 eq) was added at $25-30^{\circ} \mathrm{C}$ and stirred for $3 \mathrm{~h}$. After completion of reaction, water $(300 \mathrm{~mL})$ was added and stirred. The layers were separated, organic layer washed with $1 \%$ dil. aqs. $\mathrm{HCl}$ solution $(75 \mathrm{~mL})$ and water $(100 \mathrm{~mL})$. Organic layer was dried over sodium sulphate and solvent was removed in vacuum to obtain amide (1a) as a pale-yellow oil $(26.8 \mathrm{~g}, 89 \%)$. (1a) - Pale-yellow oil, Yield $26.8 \mathrm{~g}, 89 \%,[\alpha]_{\mathrm{D}}{ }^{20}:+12.8^{\circ}(\mathrm{c}$ $0.5, \mathrm{CH}_{2} \mathrm{Cl}_{2}$ ), (Lit. $[\alpha]_{\mathrm{D}}{ }^{20}:-13.6^{\circ}$, c $0.5, \mathrm{CH}_{2} \mathrm{Cl}_{2}$, for $(S)$ isomer). ${ }^{9}{ }^{1} \mathrm{H}$ NMR $\left(400 \mathrm{MHz}\right.$, DMSO- $\left.\mathrm{d}_{6}\right) \delta: 1.32(\mathrm{~s}, 6 \mathrm{H})$, $1.38(\mathrm{~s}, 3 \mathrm{H}), 1.73-1.81(\mathrm{~m}, 3 \mathrm{H}), 2.15-2.20(\mathrm{~m}, 1 \mathrm{H}), 3.11(\mathrm{~s}$, $3 \mathrm{H}), 3.28-3.36(\mathrm{~m}, 2 \mathrm{H}), 3.68(\mathrm{~s}, 3 \mathrm{H}), 4.53(\mathrm{dd}, J=7.2 \mathrm{~Hz}$, $1 \mathrm{H}$, mixture of rotamers). ${ }^{13} \mathrm{C}$ NMR $\left(125 \mathrm{MHz}, \mathrm{DMSO}-\mathrm{d}_{6}\right)$ : 172.9, 153.0, 78.4, 61.1, 56.2, 46.4, 32.0, 30.0, 29.1, 28.1 23.6, 22.9, IR (ATR), $\nu / \mathrm{cm}^{-1}: 1702,1635,1384,1148$; Anal. calculated for $\mathrm{C}_{12} \mathrm{H}_{22} \mathrm{~N}_{2} \mathrm{O}_{4}$ : C, 55.80; H, 8.58; N, 10.84. Found: C, $55.78 ; \mathrm{H}, 8.61 ; \mathrm{N}, 10.86$.

2.2c Synthesis of tert-butyl (R)-2-benzoylpyrrolidine1-carboxylate (1b): The solution of Amide (1a) (25g, $96 \mathrm{mmol}, 1.0 \mathrm{eq})$ in tetrahydrofuran $(150 \mathrm{~mL})$ was cooled to -45 to $-55^{\circ} \mathrm{C}$ under a nitrogen atmosphere. Added $1.0 \mathrm{M}$ $\mathrm{PhMgBr}$ solution in THF $(380 \mathrm{~mL}, 192 \mathrm{mmol}, 2.0 \mathrm{eq})$ and stirred for $1.5 \mathrm{~h}$ at -45 to $-55^{\circ} \mathrm{C}$. After completion of reaction, sat. aqs. $\mathrm{NH}_{4} \mathrm{Cl}$ solution $(300 \mathrm{~mL})$ was added and stirred. The layers were separated; aqs. layer was re-extracted with ethyl acetate $(150 \mathrm{~mL})$, the organic layer was dried over sodium sulphate and the solvent was removed in vacuum to obtain Ketone (1b) as a yellow colored oil $(22.4 \mathrm{~g}, 84 \%)$. (1b) - Yellow colored oil, Yield $22.4 \mathrm{~g}, 84 \%,[\alpha]_{\mathrm{D}}{ }^{20}:+34.2^{\circ}$ (c $0.25, \mathrm{CH}_{2} \mathrm{Cl}_{2}$ ), (Lit. $-36.8^{\circ}$, c $0.25, \mathrm{CH}_{2} \mathrm{Cl}_{2}$ for $(S)$ isomer $){ }^{9} ;{ }^{1} \mathrm{H}$ NMR $(400 \mathrm{MHz}, \mathrm{MeOD}) \delta: 1.26(\mathrm{~s}, 6 \mathrm{H}), 1.48$ $(\mathrm{s}, 3 \mathrm{H}), 1.87-1.97(\mathrm{~m}, 3 \mathrm{H}), 2.42-2.46(\mathrm{~m}, 1 \mathrm{H}), 3.49-3.60(\mathrm{~m}$, $2 \mathrm{H}), 5.36\left(\mathrm{dd}, J=8.5 \mathrm{~Hz},{ }^{1} \mathrm{H}\right.$, mixture of rotamers), $7.52-$ $7.57(\mathrm{~m}, 2 \mathrm{H}), 7.64-7.68(\mathrm{~m}, 1 \mathrm{H}), 8.03-8.05(\mathrm{~m}, 2 \mathrm{H}) .{ }^{13} \mathrm{C}$
NMR (125 MHz, MeOD): 186.2, 156.1, 136.2, 134.7, 129.9, 129.8, 129.6, 129.4, 81.2, 62.7, 31.8, 28.7, 28.4, 25.1, 24.5.; IR (neat), $v / \mathrm{cm}^{-1} 1699,1621,1430,716$. Anal. calculated for $\mathrm{C}_{16} \mathrm{H}_{21} \mathrm{NO}_{3}$ : C, 69.79; H, 7.69; N, 5.09. Found: C, 69.76; $\mathrm{H}, 7.71 ; \mathrm{N}, 5.08$.

\section{2d Synthesis of (1R,2R)-syn-1-Phenyl-2-pyrrol} idinemethanol (1c): The solution of Ketone (1b) $(25 \mathrm{~g}$, $91 \mathrm{mmol}, 1.0 \mathrm{eq})$ in ethyl acetate $(125 \mathrm{~mL})$ was cooled to $0-10^{\circ} \mathrm{C}$. Added $30 \% \mathrm{HCl}$ solution $(333 \mathrm{~mL}, 273 \mathrm{mmol}$, $3.0 \mathrm{eq}$ ) and stirred for $1.5 \mathrm{~h}$ at $0-20^{\circ} \mathrm{C}$. After completion of reaction, the $\mathrm{pH}$ was adjusted to 8 by aqs $\mathrm{NH}_{4} \mathrm{OH}$ solution and stirred. The layers were separated and aqs layers re-extracted with ethyl acetate $(125 \mathrm{~mL})$. The organic layer was dried over sodium sulphate and the solvent was removed in vacuum to get an oil. To oil charged methanol $(250 \mathrm{~mL}), \mathrm{NaBH}_{4}$ $(6.9 \mathrm{~g}, 182 \mathrm{mmol}, 2.0 \mathrm{eq})$ was added and stirred at $10-20^{\circ} \mathrm{C}$ for $1.5 \mathrm{~h}$. After completion of reaction, the solvent evaporated under vacuum to get the residue. To residue, charged water $(250 \mathrm{~mL})$, ethyl acetate $(250 \mathrm{~mL})$ and stirred. The layers were separated, aqs layer re-extracted with ethyl acetate $(100 \mathrm{~mL})$. Organic layer dried over sodium sulphate and the solvent removed in vacuum to get an oil. The $(1 R, 2 R)$ syn-1-Phenyl-2-pyrrolidinemethanol (1c) was isolated from oil by purifying it with column chromatography (eluent-5\% methanol in dichloromethane). Brown colored oil, (10.94 g, $68 \%)$.

(1c) - Brown colored oil, Yield $10.94 \mathrm{~g}, 68 \%,[\alpha]_{\mathrm{D}}-41.1^{\circ}$ (c 1.0 in $\mathrm{CHCl}_{3}$ ), (Lit. $-43.2^{\circ}$, c $1.0, \mathrm{CHCl}_{3}$ ) ${ }^{10} ;{ }^{1} \mathrm{H}$ NMR

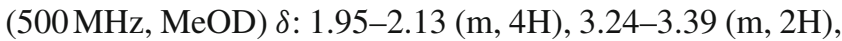
$3.72-3.87(\mathrm{~m}, 1 \mathrm{H}), 4.73-4.91(\mathrm{dd}, J=7.2 \mathrm{~Hz}, 1 \mathrm{H}), 7.33-$ $7.48(\mathrm{~m}, 5 \mathrm{H}) ;{ }^{13} \mathrm{C}$ NMR $(125 \mathrm{MHz}, \mathrm{MeOD}) 139.9,128.6$, 128.7, 128.4, 127.6, 126.8, 74.1, 66.1, 45.2, 27.6, 24.9, 24.0; IR (ATR), $v / \mathrm{cm}^{-1} 3500,3359,1402,710$; Anal. calculated for $\mathrm{C}_{11} \mathrm{H}_{15} \mathrm{NO}$ : C, 74.54; H, 8.53; N, 7.90. Found: C, 74.58; $\mathrm{H}, 8.51 ; \mathrm{N}, 7.88$.

\section{2e Synthesis of (R)-1-((R)-hydroxy(phenyl)methyl)-} 5-azaspiro [4.4] nonan-5-ium bromide (1d): To a solution of Amine (1c) $(25 \mathrm{~g}, 141 \mathrm{mmol}, 1.0 \mathrm{eq})$ in acetonitrile $(200 \mathrm{~mL})$, were charged $\mathrm{K}_{2} \mathrm{CO}_{3}(38.92 \mathrm{~g}, 282 \mathrm{mmol}, 2.0 \mathrm{eq})$ and 1,4 dibromo butane $(33.50 \mathrm{~g}, 155 \mathrm{mmol}, 1.1 \mathrm{eq})$. The reaction mass was heated to reflux and stirred for $12 \mathrm{~h}$. After completion of reaction, it was cooled and filtered, evaporated the solvent from filtrate under vacuum to get crude material, which was recrystallized by using acetone $(125 \mathrm{~mL})$ to get pure (R)-1-((R)-hydroxy(phenyl)methyl)-5-azaspiro [4.4] nonan-5-ium bromide (1d) as an off white solid, $(37.87 \mathrm{~g}$, $86 \%)$.

(1d) - White solid, Yield $37.87 \mathrm{~g}, 86 \%$, M.p.: more than $238^{\circ} \mathrm{C},[\alpha]_{\mathrm{D}}{ }^{20}-17.96^{\circ}$ (c 1.0 in Methanol); ${ }^{1} \mathrm{H}$ NMR $\left(500 \mathrm{MHz}, \mathrm{DMSOd}_{6}\right) \delta: 1.39-1.76(\mathrm{~m}, 2 \mathrm{H}), 1.88-2.17(\mathrm{~m}$, $6 \mathrm{H}), 3.48-3.76(\mathrm{~m}, 5 \mathrm{H}), 4.11-4.23(\mathrm{~m}, 2 \mathrm{H}), 4.95-4.99(\mathrm{dd}$, $J=3.2 \mathrm{~Hz}, 1 \mathrm{H}), 6.18\left(\mathrm{~s},{ }^{1} \mathrm{H}, \mathrm{D}_{2} \mathrm{O}\right.$ exchangeable $), 7.28-7.50$ $(\mathrm{m}, 5 \mathrm{H}) ;{ }^{13} \mathrm{C}$ NMR $\left(125 \mathrm{MHz}, \mathrm{DMSOd}_{6}\right) \delta: 141.8,128.4$, 128.3, 128.1, 127.4, 127.1 74.9, 72.0, 64.8, 64.3, 62.9,21.3, 20.6, 20.5, 20.3; IR (ATR), v / $\mathrm{cm}^{-1} 3143,3009,1490,704$; 
Anal. calculated for $\mathrm{C}_{15} \mathrm{H}_{22} \mathrm{NOBr}$ C, $57.70 ; \mathrm{H}, 7.10 ; \mathrm{N}, 4.49$. Found: C, 57.64; H, 7.08; N, 4.50.

$2.2 \mathrm{f}$ Synthesis of $(R)-1-((R)-h y d r o x y($ phenyl)methyl)5-azaspiro [4,5] decan-5-ium bromide (1e): To a solution of Amine (1c) $(25 \mathrm{~g}, 141 \mathrm{mmol}, 1.0 \mathrm{eq})$ in acetonitrile $\left(200 \mathrm{~mL}\right.$ ), were charged $\mathrm{K}_{2} \mathrm{CO}_{3}(38.92 \mathrm{~g}, 282 \mathrm{mmol}, 2.0 \mathrm{eq})$ and 1,5 dibromo pentane $(35.66 \mathrm{~g}, 155 \mathrm{mmol}, 1.1 \mathrm{eq})$ reaction mass was heated to reflux and stirred for $12 \mathrm{~h}$. After completion of reaction, it was cooled and filtered, evaporated the solvent from filtrate under vacuum to get crude material, which was recrystallized by using acetone $(125 \mathrm{~mL})$ to get pure (R)-1-((R)-hydroxy(phenyl)methyl)-5-azaspiro [4.4] nonan-5-ium bromide (1e) as an off white colored solid, $(38.19 \mathrm{~g}, 83 \%)$.

(1e) - White solid, Yield $38.19 \mathrm{~g}, 83 \%$, M.p.: more than $238^{\circ} \mathrm{C},[\alpha]_{\mathrm{D}}{ }^{20}-22.22^{\circ}$ (c 1.0 in Methanol); ${ }^{1} \mathrm{H}$ NMR (500 $\left.\mathrm{MHz} \mathrm{DMSOd}_{6}\right) \delta: 1.27-1.45(\mathrm{~m}, 2 \mathrm{H}), 1.69-2.08(\mathrm{~m}, 8 \mathrm{H})$, $3.36-4.19(\mathrm{~m}, 7 \mathrm{H}), 4.98-4.99(\mathrm{dd}, J=2.0 \mathrm{~Hz}, 1 \mathrm{H})$, $6.30\left(\mathrm{~s},{ }^{1} \mathrm{H}, \mathrm{D}_{2} \mathrm{O}\right.$ exchangeable), $7.29-7.48(\mathrm{~m}, 5 \mathrm{H}) ;{ }^{13} \mathrm{C}$ NMR $\left(125 \mathrm{MHz}, \mathrm{DMSOd}_{6}\right) \delta$ : 141.8, 128.3, 127.2, 126.1, 79.5, 71.6, 62.5, 59.3, 51.1, 25.4, 21.7, 21.4,19.7, 19.1; IR (neat), $v / \mathrm{cm}^{-1} 3183,2948,1493,708$. Anal. calculated for $\mathrm{C}_{16} \mathrm{H}_{24} \mathrm{NOBr}$ : C, 58.90; H, 7.41; N, 4.29. Found; C, 58.98; $\mathrm{H}, 7.38 ; \mathrm{N}, 4.28$.

\subsection{Synthesis of chiral PTCs from mandelic acid}

2.3a Synthesis of (R)-2-hydroxy-1,2-diphenylethan-1one (2): R-mandelic acid $(25 \mathrm{~g}, 164 \mathrm{mmol}, 1.0 \mathrm{eq})$ was charged in dichloromethane $(200 \mathrm{~mL})$, followed by charging of 1,1'-Carbonyl diimidazole $(32 \mathrm{~g}, 197 \mathrm{mmol}, 1.2 \mathrm{eq})$ and stirred for $0.5 \mathrm{~h}$. N, O-dimethyl hydroxylamine hydrochloride $(19.24 \mathrm{~g}, 197 \mathrm{mmol}, 1.2 \mathrm{eq})$ was added at $25-30^{\circ} \mathrm{C}$ and stirred for $2 \mathrm{~h}$. After completion of reaction water $(300 \mathrm{~mL})$ was added and stirred. The layers were separated, the organic layer washed with $1 \%$ dil. aqs $\mathrm{HCl}$ solution $(75 \mathrm{~mL})$ and water $(100 \mathrm{~mL})$. Organic layer dried over sodium sulphate and the solvent removed in vacuum to obtain the amide as a colorless oil. Tetrahydrofuran $(150 \mathrm{~mL})$ under a nitrogen atmosphere was charged to oil and cooled the reaction mass to -55 to $-65^{\circ} \mathrm{C}$. Added 1.0 M Phenyl magnesium bromide solution in THF $(812 \mathrm{~mL}, 410 \mathrm{mmol}, 2.5 \mathrm{eq})$ and stirred for $2 \mathrm{~h}$ at -55 to $-65^{\circ} \mathrm{C}$. After completion of reaction, sat. aqs $\mathrm{NH}_{4} \mathrm{Cl}$ solution $(300 \mathrm{~mL})$ was added and stirred. The layers were separated, aqs layers re-extracted with ethyl acetate $(150 \mathrm{~mL})$, Organic layer dried over sodium sulphate and the solvent removed in vacuum to obtain crude product, which was purified by column chromatography (eluent $12 \%$ ethyl acetate in n-hexane) to obtain the (R)-2-hydroxy-1,2-diphenylethan-1-one (2) as white solid $(23.83 \mathrm{~g}, 67 \%)$.

(2) - White solid, Yield 21.19 g, $78 \%$, M.p.: $133.2-134.7^{\circ} \mathrm{C}$ (Lit. $\left.135^{\circ} \mathrm{C}\right)^{11} ;[\alpha]_{\mathrm{D}}{ }^{22}:-107.9^{\circ}$ (c 1.5, acetone), (Lit. $-112.1^{\circ}, c 1.5$, acetone $){ }^{11} ;{ }^{1} \mathrm{H}$ NMR (500 MHz, DMSO-d 6 ) $\delta: 6.12(\mathrm{~s}, 1 \mathrm{H}), 6.14\left(\mathrm{~s},{ }^{1} \mathrm{H}\right.$, exchangeable with $\left.\mathrm{D}_{2} \mathrm{O}\right)$, $7.21-7.25\left(\mathrm{~m},{ }^{1} \mathrm{H}\right), 7.32(\mathrm{t}, J=8.0,2 \mathrm{H}), 7.43-7.48(\mathrm{~m}, 4 \mathrm{H})$, $7.53-7.56\left(\mathrm{~m},{ }^{1} \mathrm{H}\right), 8.03(\mathrm{dd}, J=8.0,1.5 \mathrm{~Hz}, 2 \mathrm{H}) ;{ }^{13} \mathrm{C} \mathrm{NMR}$
(125 MHz, DMSO-d $\left.\mathrm{d}_{6}\right): 199.2,139.7,134.7,133.2,128.8$, 128.6, 128.5, 127.7, 127.3, 75.7; IR (ATR), $v / \mathrm{cm}^{-1} 3466$, 1728, 1449, 1130, 1089, 731; Anal. calculated for $\mathrm{C}_{14} \mathrm{H}_{12} \mathrm{O}_{2}$ : C, 79.23; H, 5.70. Found: C, 79.17; H, 5.69.

2.3b Synthesis of (1R,2R)-2-(methylamino)-1,2diphenylethan-1-ol (2a): Ketone (2) $(25 \mathrm{~g}, 117 \mathrm{mmol}$, 1.0 eq) was charged to $2 \mathrm{M}$ methylamine solution in methanol $(500 \mathrm{~mL}, 1.0 \mathrm{~mol}, 8.54 \mathrm{eq})$ at $0-10^{\circ} \mathrm{C}$ and stirred for $6 \mathrm{~h}$. After completion of reaction, $\mathrm{NaBH}_{4}(8.85 \mathrm{~g}, 234 \mathrm{mmol}$, $2.0 \mathrm{eq}$ ) was added and stirred for $2 \mathrm{~h}$ at $0-10^{\circ} \mathrm{C}$. After completion of the reaction, the solvent evaporated under vacuum to get the residue. To the residue, water $(500 \mathrm{~mL})$ and ethyl acetate $(500 \mathrm{~mL})$ were charged and the mixture was stirred. The layers were separated and the aqs. layer was re-extracted with ethyl acetate $(100 \mathrm{~mL})$. The organic layer was dried over sodium sulphate and the solvent was removed in vacuum to obtain crude solid product, which was purified by recrystallization in methanol to get $(1 \mathrm{R}, 2 \mathrm{R})$ 2-(methylamino)-1,2-diphenylethan-1-ol (2a) as white solid $(23.29 \mathrm{~g}, 87 \%)$.

(2a) - White solid, Yield 23.29g, 87\%, M.p.: 119.3$1121.1^{\circ} \mathrm{C}, \quad[\alpha]_{\mathrm{D}}{ }^{25}: 36.2^{\circ}$ (c 1.0 methanol); ${ }^{1} \mathrm{H}$ NMR $\left(500 \mathrm{MHz}\right.$, DMSO- $\left.\mathrm{d}_{6}\right) \delta: 1.77\left(\mathrm{~s},{ }^{1} \mathrm{H}\right.$, exchangeable with $\left.\mathrm{D}_{2} \mathrm{O}\right), 2.05(\mathrm{~s}, 3 \mathrm{H}), 3.62(\mathrm{~d}, J=5.5 \mathrm{~Hz}, 1 \mathrm{H}), 4.68(\mathrm{~d}$, $J=5.0 \mathrm{~Hz}, 1 \mathrm{H}), 5.28(\mathrm{~s}, 1 \mathrm{H}), 7.15-7.24(\mathrm{~m}, 10 \mathrm{H}) ;{ }^{13} \mathrm{C} \mathrm{NMR}$ $\left(125 \mathrm{MHz}\right.$, DMSO-d $\left.\mathrm{d}_{6}\right): 143.2,140.8,128.5,127.4,127.3$, 126.9, 126.7, 126.4, 76.2, 70.6, 34.1; IR (ATR), $v / \mathrm{cm}^{-1}$ 3318, 3245, 1490, 1467, 744, 697. Anal. calculated for $\mathrm{C}_{15} \mathrm{H}_{17} \mathrm{NO}$ : C, 79.26; H, 7.54; N, 6.16. Found: C, 79.21; H, $7.55 ; \mathrm{N}, 6.18$.

\section{3c Synthesis of 1-((1R,2R)-2-hydroxy-1,2-dip} henylethyl)-1-methylpyrrolidin-1-ium bromide $(\mathbf{2 b})$ :

To a solution of amine (2a) $(25 \mathrm{~g}, 110 \mathrm{mmol}, 1.0 \mathrm{eq})$ in acetonitrile $(200 \mathrm{~mL})$, were charged $\mathrm{K}_{2} \mathrm{CO}_{3}(30.40 \mathrm{~g}, 220 \mathrm{mmol}$, $2.0 \mathrm{eq})$ and 1,4 dibromo butane $(26.12 \mathrm{~g}, 121 \mathrm{mmol}, 1.1 \mathrm{eq})$. The reaction mass heated to reflux and stirred for $12 \mathrm{~h}$. After completion of reaction, it was cooled and filtered. The solvent was evaporated from filtrate under vacuum to get crude material, which was recrystallized by using acetone $(125 \mathrm{~mL})$ to get pure 1-((1R,2R)-2-hydroxy-1,2diphenylethyl)-1-methylpyrrolidin-1-ium bromide (2b) as a white colored solid, (32.66 g, 82\%).

(2b) - White solid, Yield $32.66 \mathrm{~g}, 82 \%$, M.p.: more than $238^{\circ} \mathrm{C},[\alpha]_{\mathrm{D}}{ }^{20} 21.38^{\circ}$ (c 1.0 in Methanol); ${ }^{1} \mathrm{H}$ NMR $\left(500 \mathrm{MHz}, \mathrm{DMSO}_{\mathrm{d}} \mathrm{d}_{6}\right) \delta: 2.02-2.07(\mathrm{~m}, 2 \mathrm{H}), 2.15-2.21(\mathrm{~m}$, $2 \mathrm{H}), 3.04-3.08\left(\mathrm{~m},{ }^{1} \mathrm{H}\right), 3.20(\mathrm{~s}, 3 \mathrm{H}), 3.70-3.76 .\left(\mathrm{m},{ }^{1} \mathrm{H}\right)$, $3.85-3.94(\mathrm{~m}, 2 \mathrm{H}), 4.83\left(\mathrm{~d}, J=2.5 \mathrm{~Hz},{ }^{1} \mathrm{H}\right), 5.76(\mathrm{~d}$, $J=2.0 \mathrm{~Hz}, 1 \mathrm{H}), 6.53\left(\mathrm{~s}, 1 \mathrm{H}, \mathrm{D}_{2} \mathrm{O}\right.$ exchangeable), 7.06-7.59 $(\mathrm{m}, 10 \mathrm{H}) ;{ }^{13} \mathrm{C}$ NMR $(125 \mathrm{MHz}$, DMSO-d 6 ) $\delta: 141.1,132.6$, 129.9, 129.6, 128.1, 127.6, 127.06, 126.1, 78.5, 70.8, 64.0, 62.7, 45.9, 20.5, 20.3; IR (ATR), v / $\mathrm{cm}^{-1} 3418,3072,1476$, 1484, 728, 706. Anal. calculated for $\mathrm{C}_{19} \mathrm{H}_{24} \mathrm{NOBr}$ : C, 62.99; H, 6.68; N, 3.87. Found; C, 62.94; H, 6.70; N, 3.88 . 
2.3d Synthesis of 1-((1R,2R)-2-hydroxy-1,2diphenylethyl)-1-methylazepan-1-ium bromide (2c): To a solution of amine (2b) $(25 \mathrm{~g}, 110 \mathrm{mmol}, 1.0 \mathrm{eq})$ in acetonitrile $(200 \mathrm{~mL})$, were charged $\mathrm{K}_{2} \mathrm{CO}_{3}(30.40 \mathrm{~g}, 220 \mathrm{mmol}$, $2.0 \mathrm{eq})$ and 1,6 dibromo hexane $(29.55 \mathrm{~g}, 121 \mathrm{mmol}, 1.1 \mathrm{eq})$. The reaction mixture was heated to reflux and stirred for $12 \mathrm{~h}$. After completion of reaction, it was cooled and filtered. The solvent was evaporated from filtrate under vacuum to get crude material, which was recrystallized by using acetone $(125 \mathrm{~mL})$ to get pure 1-((1R,2R)-2-hydroxy1,2-diphenylethyl)-1-methylazepan-1-ium bromide (2c) as a white colored solid, (31.81 g, 74\%).

(2c) - White solid, Yield 31.81g, 74\%, M.p.: more than $238^{\circ} \mathrm{C},[\alpha]_{\mathrm{D}}{ }^{20} 26.23^{\circ}$ (c 1.0 in Methanol); ${ }^{1} \mathrm{H}$ NMR $\left(500 \mathrm{MHz}, \mathrm{DMSO}-\mathrm{d}_{6-}\right.$ very less solubility) $\delta: 1.52-1.94(\mathrm{~m}$, $8 \mathrm{H}), 3.21$ (s, 3H), 3.64-4.16 (m, 4H), 4.62 (d, $J=2.5 \mathrm{~Hz}$, $1 \mathrm{H}), 5.81(\mathrm{~d}, J=2.0 \mathrm{~Hz}, 1 \mathrm{H}), 6.58\left(\mathrm{~s}, 1 \mathrm{H}, \mathrm{D}_{2} \mathrm{O}\right.$ exchangeable), 6.92-8.17 (m, 10H); ${ }^{13} \mathrm{C}$ NMR (125 MHz DMSO-d very less solubility) $\delta$ : 129.1, 128.9, 128.8, 128.4, 128.3, 127.4, 127.2, 82.5,74.4, 72.2, 71.8, 71.1, 66.3, 65.6, 64.4, 55.6,28.7, 27.1, 23.4, 23.3; IR (ATR), $v / \mathrm{cm}^{-1} 3422,3061$, 1480, 1492, 730, 707. Anal. calculated for $\mathrm{C}_{21} \mathrm{H}_{28} \mathrm{NOBr}$ : C, $64.61 ; \mathrm{H}, 7.23 ; \mathrm{N}, 3.59$. Found; C, 64.55; H, 7.21; N, 3.60.

\subsection{Synthesis of chiral PTCs from tartaric acid}

2.4a Synthesis of ((4R,5R)-2,2-dimethyl-1,3-dioxolane-4,5-diyl) bis (pyrrolidin-1-ylmethanone) (3a):

Protected diethyl tartrate (3) $(25 \mathrm{~g}, 101 \mathrm{mmol}, 1.0 \mathrm{eq})$ was charged to pyrrolidine $(50 \mathrm{~mL}, 606 \mathrm{mmol}, 6.0 \mathrm{eq})$ and stirred for $10 \mathrm{~h}$ at $20-30^{\circ} \mathrm{C}$. After completion of reaction, the reaction mixture was filtered to get crude material, which was recrystallized by using acetone $(125 \mathrm{~mL})$ to get pure (3a) as a yellow colored solid, $(27.4 \mathrm{~g}, 91 \%)$.

(3a) - Yellow solid, Yield 27.4g, 91\%, M.p.: $112.8-113.5^{\circ} \mathrm{C},[\alpha]_{\mathrm{D}}{ }^{20}-47.72$ (c 1.0 in Methanol); ${ }^{1} \mathrm{H}$ NMR $\left(500 \mathrm{MHz}, \mathrm{DMSOd}_{6}\right) \delta: 1.36(\mathrm{~s}, 6 \mathrm{H}), 1.76-1.80(\mathrm{~m}, 4 \mathrm{H})$, 1.83-1.89 (m, 4H), 3.30-3.33 (m, 4H), 3.54-3.56 (m, 4H), $4.98(\mathrm{~s}, 2 \mathrm{H}) ;{ }^{13} \mathrm{C}$ NMR $\left(125 \mathrm{MHz}, \mathrm{DMSOd}_{6}\right) \delta: 166.2,111.3$, 76.13, 45.9, 45.8, 26.1, 25.5, 23.4; IR (ATR), $\nu / \mathrm{cm}^{-1} 2958$, 1440, 1126, 850, 735. Anal. calculated for $\mathrm{C}_{15} \mathrm{H}_{24} \mathrm{~N}_{2} \mathrm{O}_{4}$ : C, $60.79 ; \mathrm{H}, 8.16 ; \mathrm{N}, 9.45$. Found; C, 60.85; H, 8.15; N, 9.43.

2.4b Synthesis of ((4R,5R)-2,2-dimethyl-1,3dioxolane-4,5-diyl) bis (piperidin-1-ylmethanone) $(3 \boldsymbol{b})$ : Protected diethyl tartrate (3) $(25 \mathrm{~g}, 101 \mathrm{mmol}, 1.0 \mathrm{eq})$ was charged to piperazine $(60 \mathrm{~mL}, 606 \mathrm{mmol}, 6.0 \mathrm{eq})$ and stirred for $10 \mathrm{~h}$ at $20-30^{\circ} \mathrm{C}$. After completion of reaction, the reaction mass was filtered to get crude material, which was recrystallized by using acetone $(125 \mathrm{~mL})$ to get pure $((4 \mathrm{R}, 5 \mathrm{R})-2,2-$ dimethyl-1,3-dioxolane-4,5-diyl) bis (piperidin-1 -ylmethanone) (3b) as a yellow colored solid, (29.3 g, 89\%).

(3b) - Yellow solid, Yield 29.3g, 89\%, M.p.: $115.3-118.1{ }^{\circ} \mathrm{C},[\alpha]_{\mathrm{D}}{ }^{20}-24.30$ (c 1.0 in Methanol); ${ }^{1} \mathrm{H}$ NMR $\left(500 \mathrm{MHz}, \mathrm{DMSOd}_{6}\right) \delta: 1.35(\mathrm{~s}, 6 \mathrm{H}), 1.42-1.47(\mathrm{~m}, 4 \mathrm{H})$, $1.48-1.54(\mathrm{~m}, 4 \mathrm{H}), 1.58-1.62(\mathrm{~m}, 4 \mathrm{H}), 3.33(\mathrm{~m}, 2 \mathrm{H}), 3.47-$ $3.58(\mathrm{~m}, 6 \mathrm{H}), 5.15(\mathrm{~s}, 2 \mathrm{H}) ;{ }^{13} \mathrm{C} \mathrm{NMR}\left(125 \mathrm{MHz}, \mathrm{DMSOd}_{6}\right)$ $\delta: 165.8,111.1,74.7,45.8,42.5,26.2,26.0,25.2,23.9 ;$ IR (ATR), $v / \mathrm{cm}^{-1} 2950,1444,1132,854,732$. Anal. calculated for $\mathrm{C}_{17} \mathrm{H}_{28} \mathrm{~N}_{2} \mathrm{O}_{4}$ : C, 62.94; H, 8.70; N, 8.64. Found; C, $63.01 ; \mathrm{H}, 8.69 ; \mathrm{N}, 8.62$.

\section{4c Synthesis of 1,1'-(((4R,5R)-2,2-dimethyl-1,3} -dioxolane-4,5-diyl) bis (methylene)) dipyrrolidine (3c): The solution of Amide (3a) $(25 \mathrm{~g}, 84 \mathrm{mmol}, 1.0 \mathrm{eq})$ in 1,4 dioxane $(250 \mathrm{~mL})$ was added slowly to a suspension of LAH $(9.56 \mathrm{~g}, 252 \mathrm{mmol}, 3.0 \mathrm{eq})$ in dioxane $(300 \mathrm{~mL})$ under nitrogen and refluxed for $12 \mathrm{~h}$. After completion of reaction, the reaction mass was cooled and quenched with water $(25 \mathrm{~mL}), 15 \%$ aqs $\mathrm{NaOH}$ solution $(25 \mathrm{~mL})$ and water $(25 \mathrm{~mL})$ were added. The reaction mass was stirred and filtered. The solvent was evaporated from the filtrate under vacuum to get $(\mathbf{3 c})$, as a pale brown colored oil $(21.05 \mathrm{~g}, 93 \%)$.

(3c) - Pale brown colored oil, Yield $21.05 \mathrm{~g}, 93 \%,[\alpha]_{\mathrm{D}}{ }^{20}$ -40.36 (c 1.0 in Methanol); ${ }^{1} \mathrm{H}$ NMR $\left(500 \mathrm{MHz}, \mathrm{CDCl}_{3}\right) \delta$ : $1.21(\mathrm{~s}, 6 \mathrm{H}), 1.55-1.58(\mathrm{~m}, 8 \mathrm{H}), 2.34-2.46(\mathrm{~m}, 12 \mathrm{H}), 3.55$ 3.61(m, $2 \mathrm{H}) ;{ }^{13} \mathrm{C}$ NMR $\left.\left(125 \mathrm{MHz} \mathrm{CDCl}_{3}\right)\right) \delta: 108.9,77.6$, 72.6, 72.5, 58.8, 54.6, 27.0, 23.2; IR (ATR), $v / \mathrm{cm}^{-1} 2965$, 2784, 1460, 1434, 1378, 1368, 1239, 1156, 1066, 988, 882, 849, 672. Anal. calculated for $\mathrm{C}_{15} \mathrm{H}_{28} \mathrm{~N}_{2} \mathrm{O}_{2}: \mathrm{C}, 67.13 ; \mathrm{H}$, $10.52 ; \mathrm{N}, 10.44$. Found; C, 67.17; H, 10.50; N, 10.43 .

\section{4d Synthesis of 1,1'-(((4R,5R)-2,2-dimethyl-1,3} -dioxolane-4,5-diyl) bis (methylene)) dipiperidine (3d): The solution of Amide (3b) $(25 \mathrm{~g}, 77 \mathrm{mmol}, 1.0 \mathrm{eq})$ in 1,4 dioxane $(250 \mathrm{~mL})$ was added slowly to a suspension of LAH $(8.77 \mathrm{~g}, 231 \mathrm{mmol}, 3.0 \mathrm{eq})$ in dioxane $(300 \mathrm{~mL})$ under nitrogen and refluxed for $12 \mathrm{~h}$. After completion of reaction, the reaction mass was cooled and quenched with water $(25 \mathrm{~mL}), 15 \%$ aqs $\mathrm{NaOH}$ solution $(25 \mathrm{~mL})$ and water $(25 \mathrm{~mL})$ were added. The reaction mass was stirred and filtered. The solvent was evaporated from filtrate under vacuum to get 1,1'-(((4R,5R)-2,2-dimethyl-1,3-dioxolane-4,5-diyl) bis (methylene)) dipiperidine (3d) as a pale brown colored oil, (20.56 g, 90\%).

(3d) - Pale brown colored oil, Yield $20.56 \mathrm{~g}, 90 \%,[\alpha]_{\mathrm{D}}{ }^{20}$ -27.80 (c1.0 in methanol); ${ }^{1} \mathrm{H}$ NMR $\left(500 \mathrm{MHz}, \mathrm{CDCl}_{3}\right) \delta$ : $1.22(\mathrm{~s}, 6 \mathrm{H}), 1.24-1.27(\mathrm{~m}, 4 \mathrm{H}), 1.41-1.45(\mathrm{~m}, 8 \mathrm{H}), 2.27-2.38$ (m, 12H), 3.68-3.69(m, 2H); ${ }^{13} \mathrm{C} \mathrm{NMR} \mathrm{(125} \mathrm{MHz} \mathrm{CDCl} 3$ ) $\delta$ : 108.8, 77.8, 61.9, 55.2, 27.1, 25.7, 24.1; IR (ATR), $v / \mathrm{cm}^{-1}$ 3050, 1126, 742, 698. Anal. calculated for $\mathrm{C}_{17} \mathrm{H}_{32} \mathrm{~N}_{2} \mathrm{O}_{2}$ : C, $68.88 ; \mathrm{H}, 10.88 ; \mathrm{N}, 9.45$. Found; C, 68.90; H, 10.86; N, 9.44.

\section{$2.4 \mathrm{e}$ Synthesis of 1,1'-(((4R,5R)-2,2-dimethyl-1,3} -dioxolane-4,5-diyl) bis (methylene)) bis (1-benzyl pyrrolidin-1-ium) bromide (3e): To a solution of Amine (3c) $(25 \mathrm{~g}, 93 \mathrm{mmol}, 1.0 \mathrm{eq})$ in acetonitrile $(200 \mathrm{~mL})$, were charged $\mathrm{K}_{2} \mathrm{CO}_{3}(38.55 \mathrm{~g}, 279 \mathrm{mmol}, 3.0 \mathrm{eq})$ and benzyl bromide $(34.99 \mathrm{~g}, 204 \mathrm{mmol}, 2.2 \mathrm{eq})$. The reaction mixture was heated to reflux and stirred for $10 \mathrm{~h}$. After completion of reaction, the reaction mass was cooled and filtered. The filtrate was evaporated under vacuum to get crude material, which 
was recrystallized by using acetone $(150 \mathrm{~mL})$ to get pure $1,1^{\prime}-$ (((4R,5R)-2,2-dimethyl-1,3-dioxolane-4,5-diyl) bis (methylene)) bis (1-benzylpyrrolidin-1-ium) bromide (3e) as a white colored solid, (50.03 g, 88\%).

(3e) - White solid, Yield 50.03 g, 88\%, M.p.: more than $238^{\circ} \mathrm{C},[\alpha]_{\mathrm{D}}{ }^{20}-15.51$ (c 1.0 in Methanol); ${ }^{1} \mathrm{H}$ NMR $\left(500 \mathrm{MHz}, \mathrm{CDCl}_{3}\right) \delta: 1.55(\mathrm{~s}, 6 \mathrm{H}), 2.07-2.15(\mathrm{~m}, 8 \mathrm{H}), 3.55-$ $3.56(\mathrm{~m}, 4 \mathrm{H}), 3.64-3.3 .73(\mathrm{~m}, 6 \mathrm{H}), 3.88-3.91(\mathrm{~m}, 2 \mathrm{H})$, 4.60-4.64 (m, 2H), 4.67-4.73 (m, 4H), 7.50-7.69 (m, 10H); ${ }^{13} \mathrm{C}$ NMR $\left(125 \mathrm{MHz}, \mathrm{CDCl}_{3}\right) \delta: 133.0,130.4,129.1,128.1$, 127.5, 127.0, 73.6, 72.5, 64.3, 63.6, 61.3, 61.2, 27.2, 21.3; IR (ATR), $v / \mathrm{cm}^{-1} 3189,2946,1494,1452,1142,1118,740$, 710. Anal. calculated for $\mathrm{C}_{29} \mathrm{H}_{42} \mathrm{~N}_{2} \mathrm{O}_{2} \mathrm{Br}_{2}$ : C, $57.06 ; \mathrm{H}, 6.93$; N, 4.59. Found; C, 57.11; H, 6.91; N4.58.

2.4f Synthesis of 1,1'-(( $4 R, 5 R)-2,2$-dimethyl-1, 3-dioxolane-4,5-diyl) bis (methylene)) bis (1-ben zylpiperidin-1-ium) bromide (3f): To a solution of Amine (3d) $(25 \mathrm{~g}, 84 \mathrm{mmol}, 1.0 \mathrm{eq})$ in acetonitrile $(200 \mathrm{~mL})$, were charged $\mathrm{K}_{2} \mathrm{CO}_{3}(34.77 \mathrm{~g}, 252 \mathrm{mmol}, 3.0 \mathrm{eq})$ and 1,5 dibromo pentane $(31.60 \mathrm{~g}, 184 \mathrm{mmol}, 2.2 \mathrm{eq})$. The reaction mass was heated to reflux and stirred for $10 \mathrm{~h}$. After completion of reaction, the reaction mass was cooled and filtered. The filtrate was evaporated under vacuum to get crude material, which was recrystallized by using acetone $(150 \mathrm{~mL})$ to get pure 1,1'-(((4R,5R)-2,2-dimethyl-1,3-dioxolane-4,5diyl) bis (methylene)) bis (1-benzylpiperidin-1-ium) bromide (3f) as a white colored solid, (49.0 g, 91\%).

(3f) - White solid, Yield 49.0g, 91\%, M.p.: more than $238^{\circ} \mathrm{C},[\alpha]_{\mathrm{D}}{ }^{20}-15.3$ (c 1.0 in methanol); ${ }^{1} \mathrm{H}$ NMR $(500 \mathrm{MHz}$, DMSOd $\left._{6}\right) \delta: 1.41-1.44(\mathrm{~m}, 4 \mathrm{H}), 1.55(\mathrm{~s}, 6 \mathrm{H}), 1.62-2.08(\mathrm{~m}$, $8 \mathrm{H}), 3.23-3.16(\mathrm{~m}, 4 \mathrm{H}), 3.53-3.56(\mathrm{~m}, 2 \mathrm{H}), 3.64-3.67(\mathrm{~m}$, $2 \mathrm{H}), 3.92-3.93(\mathrm{~m}, 2 \mathrm{H}), 4.09-4.11(\mathrm{~m}, 2 \mathrm{H}), 4.81-4.85(\mathrm{~m}$, $4 \mathrm{H}), 4.96-4.98(\mathrm{~m}, 2 \mathrm{H}), 7.49-7.70(\mathrm{~m}, 10 \mathrm{H}) ;{ }^{13} \mathrm{C} \mathrm{NMR}$ $\left(125 \mathrm{MHz}, \mathrm{DMSOd}_{6}\right) \delta: 133.5,130.3,128.9,128.0,127.5$, 112.0, 71.9, 64.2, 58.2, 57.9, 26.8, 20.4, 19.2; IR (ATR), $v / \mathrm{cm}^{-1} 3192,2942,1497,1455,1144,1115,743,705$. Anal. calculated for $\mathrm{C}_{31} \mathrm{H}_{46} \mathrm{~N}_{2} \mathrm{O}_{2} \mathrm{Br}_{2}$ : C, 58.31; $\mathrm{H}, 7.26 ; \mathrm{N}, 4.39$. Found; C, 58.37; H, 7.28; N, 4.38 .

\subsection{General procedure for the epoxidation of enone (Chalcone) (4)}

To a solution of Chalcone $(25 \mathrm{~g}, 120 \mathrm{mmol}, 1.0 \mathrm{eq})$ in toluene $(250 \mathrm{~mL})$, was charged novel chiral PTC $(5 \mathrm{~mol} \%$ or $10 \mathrm{~mol} \%)$ followed by charging of $10 \%$ aqs sodium hypochlorite solution $(180 \mathrm{~mL}, 240 \mathrm{mmol}, 2.0 \mathrm{eq})$. The resulting mixture was stirred vigorously at $20-30^{\circ} \mathrm{C}$ for $6-12 \mathrm{~h}$. After completion of reaction, water $(500 \mathrm{~mL})$ was charged and stirred. The layers were separated, organic layer was concentrated under reduced pressure to get the crude product as a residue, which was purified by recrystallization from ethyl acetate and nhexane to get pure epoxide (4) as a white solid $(25.57 \mathrm{~g}, 95 \%)$ (Table 1).

(4) - White solid, Yield $25.57 \mathrm{~g}, 95 \%,[\alpha]_{\mathrm{D}}{ }^{24} 194.9^{\circ}(c 0.3$ in acetone). (Lit. $[\alpha]_{\mathrm{D}}{ }^{24}=201^{\circ}$ for $98 \%$ ee (c 0.3 in Acetone $){ }^{12}{ }^{1} \mathrm{H} \mathrm{NMR}\left(500 \mathrm{MHz}, \mathrm{CDCl}_{3}\right) \delta: 4.10(\mathrm{~d}, J=1.5 \mathrm{~Hz}$, $\left.{ }^{1} \mathrm{H}\right), 4.32\left(\mathrm{~d}, J=2.0 \mathrm{~Hz},{ }^{1} \mathrm{H}\right), 7.28-7.45(\mathrm{~m}, 5 \mathrm{H}), 7.51$ $(\mathrm{t}, J=7.5 \mathrm{~Hz}, 2 \mathrm{H}), 7.64\left(\mathrm{t}, J=7.0 \mathrm{~Hz},{ }^{1} \mathrm{H}\right), 8.03(\mathrm{dd}$, $J=8.5 \mathrm{~Hz}, 2 \mathrm{H}) ;{ }^{13} \mathrm{C} \mathrm{NMR}\left(125 \mathrm{MHz}, \mathrm{CDCl}_{3}\right) \delta: 193.1$, $135.5,135.4,134.0,129.1,128.9,128.8,128.3,125.8,61.0$, 59.4; IR (ATR), $v / \mathrm{cm}^{-1} 1684,1596,1575,1495,1116$, 745, 738. Anal. calculated for $\mathrm{C}_{15} \mathrm{H}_{1} 2 \mathrm{O}_{2}$ : C, 80.34; $\mathrm{H}, 5.39$. Found; C, 80.39; H, 5.37. Chiral HPLC analysis (Chiral pack, IPA: $\mathrm{n}$-Hexane $=10: 90, \lambda=254 \mathrm{~nm}$ flow rate $=1 \mathrm{~mL}$ min ${ }^{-1}$ t $_{\text {major }} \sim 7.80-7.90$ min., $t_{\text {minor }} \sim 19.20-21.30$ min. $^{13}$ (ee- 73-87\%) (Table 1).

\subsection{General procedure for the epoxide (4) synthesis} from aldehyde and $\alpha$ halo ketone

2-chloro-1-phenylethan-1-one ( $25 \mathrm{~g}, 161 \mathrm{mmol}, 1.0 \mathrm{eq})$, benzaldehyde $(20.59 \mathrm{~g}, 194 \mathrm{mmol}, 1.2 \mathrm{eq})$ were charged in round bottom flask, followed by novel chiral PTC $(5 \mathrm{~mol} \%$ or $10 \mathrm{~mol} \%)$ in tertiary butyl methyl ether $(250 \mathrm{~mL})$. The resulting mixture was cooled to $0-5^{\circ} \mathrm{C}$ and stirred for $0.5 \mathrm{~h}$. Lithium hydroxide monohydrate $(13.51 \mathrm{~g}, 322 \mathrm{mmol}, 2.0 \mathrm{eq})$ was then charged and the resulting mixture stirred vigorously for 9-18 h. After completion of reaction, it was neutralized with dil. aqs. $\mathrm{HCl}$. The layers were separated, organic layer concentrated under reduced pressure to get crude product as a residue, which was purified by recrystallization from ethyl acetate and n-hexane to get pure epoxide (4) as a white solid $(33.32 \mathrm{~g}, 91.9 \%)$ (Table 2).

(4) - White solid, Yield $33.32 \mathrm{~g}, 92 \%,[\alpha]_{\mathrm{D}}{ }^{24} 193.2^{\circ}(c 0.3$ in acetone). (Lit. $[\alpha]_{\mathrm{D}}{ }^{24}=201^{\circ}$ for $98 \%$ ee (c 0.3 in Acetone $){ }^{12} ;{ }^{1} \mathrm{H} \mathrm{NMR}\left(500 \mathrm{MHz}, \mathrm{CDCl}_{3}\right) \delta: 4.10(\mathrm{~d}, J=1.5 \mathrm{~Hz}$, $\left.{ }^{1} \mathrm{H}\right), 4.32\left(\mathrm{~d}, J=2.0 \mathrm{~Hz},{ }^{1} \mathrm{H}\right), 7.28-7.45(\mathrm{~m}, 5 \mathrm{H}), 7.53$ $(\mathrm{t}, J=7.5 \mathrm{~Hz}, 2 \mathrm{H}), 7.63\left(\mathrm{t}, J=7.0 \mathrm{~Hz},{ }^{1} \mathrm{H}\right), 8.04(\mathrm{dd}$, $J=8.5 \mathrm{~Hz}, 2 \mathrm{H}) ;{ }^{13} \mathrm{C} \mathrm{NMR}\left(125 \mathrm{MHz}, \mathrm{CDCl}_{3}\right) \delta: 193.1$, 135.5, 135.4, 134.0, 129.0, 128.9, 128.8, 128.3, 125.8, 61.0, 59.4; IR (ATR), $v / \mathrm{cm}^{-1} 1686,1594,1578,1497,1112$, 746, 740. Anal. calculated for $\mathrm{C}_{15} \mathrm{H}_{1} 2 \mathrm{O}_{2}$ : C, 80.34; $\mathrm{H}, 5.39$. Found; C, 80.29; H, 5.41. Chiral HPLC analysis (Chiral pack, IPA: $\mathrm{n}$-Hexane $=10: 90, \lambda=254 \mathrm{~nm}$ flow rate $=1 \mathrm{~mL}$ $\min ^{-1}$ ) 7.80-7.90 min., $t_{\text {minor }} \sim 19.20-21.30$ min. $^{13}$ (ee- 72 $84 \%$ ) (Table 2).

\section{Results and Discussion}

Novel quaternary ammonium salts of proline, mandelic acid and tartaric acid (1d, 1e, 2b, 2c, $\mathbf{3 e}$ and $\mathbf{3 f}$ ) are ideal because, one of the tetrahedron faces about the charged quaternary nitrogen is totally blocked by the cyclic ring system itself; the second tetrahedral face about quaternary nitrogen is blocked by the aromatic ring, whose position is fixed for steric reasons, whereas the third tetrahedral face about charged quaternary nitrogen is blocked by alkyl or benzyl or other cyclic groups. So, only one side is available for ion pairing with the reactant. The proline derivatives (1d and 1e) and mandelic acid derivatives (2b and $\mathbf{2 c}$ ) has - $\mathrm{OH}$ group and tartaric 
Table 1. Epoxidation of chalcone to get chiral epoxide (4) by using novel chiral PTCs.

\begin{tabular}{|c|c|c|c|c|c|c|}
\hline Entry & Chiral PTC & Loading of Chiral PTC ( $\mathrm{mol} \%)$ & Reaction time (h) & Yield $^{\text {a }}(\%)$ & $\operatorname{SOR}[\alpha]^{b}$ & $\mathrm{ee}^{\mathrm{c}}(2 \boldsymbol{S}, 3 \boldsymbol{R})(\%)$ \\
\hline 1 & 1d & 5 & 11 & 87 & $183.2^{\circ}$ & $78.30 \%$ \\
\hline 2 & 1d & 10 & 7 & 88 & $184.1^{\circ}$ & $78.62 \%$ \\
\hline 3 & 1e & 5 & 10 & 88 & $186.3^{\circ}$ & $79.65 \%$ \\
\hline 4 & 1e & 10 & 6 & 90 & $186.9^{\circ}$ & $79.99 \%$ \\
\hline 5 & $2 b$ & 5 & 9 & 92 & $192.1^{\circ}$ & $80.88 \%$ \\
\hline 6 & $2 b$ & 10 & 5 & 93 & $192.8^{\circ}$ & $81.23 \%$ \\
\hline 7 & $2 c$ & 5 & 9 & 94 & $194.4^{\circ}$ & $84.82 \%$ \\
\hline 8 & $2 c$ & 10 & 5 & 95 & $194.9^{\circ}$ & $87.14 \%$ \\
\hline 9 & $3 e$ & 5 & 12 & 80 & $176.6^{\circ}$ & $72.93 \%$ \\
\hline 10 & $3 e$ & 10 & 8 & 82 & $178.5^{\circ}$ & $73.29 \%$ \\
\hline 11 & $3 f$ & 5 & 11 & 82 & $178.8^{\circ}$ & $76.24 \%$ \\
\hline 12 & $3 f$ & 10 & 7 & 83 & $179.9^{\circ}$ & $76.89 \%$ \\
\hline
\end{tabular}

${ }^{a}$ Isolated yield after purification. ${ }^{\mathrm{b}} \mathrm{SOR}-\left(\mathrm{c} 0.3\right.$ in Acetone at $\left.24^{\circ} \mathrm{C}\right) .{ }^{12 \mathrm{c}}$ Enantiomeric excess $(\%$ ee $)$ for absolute configuration $(2 S, 3 R)$ was determined by HPLC analysis using a Phenomenex Chiralpack column by comparison with the HPLC retention time using known standard. ${ }^{13}$

Table 2. Darzen condensation to get chiral epoxide (4) by using novel chiral novel PTCs.

\begin{tabular}{|c|c|c|c|c|c|c|}
\hline Entry & Chiral PTC & Loading of Chiral PTC (mol \%) & Reaction time (h) & Yield $^{\text {a }}(\%)$ & $\operatorname{SOR}[\alpha]^{b}$ & $\operatorname{ee}^{\mathrm{c}}(2 S, 3 R)(\%)$ \\
\hline 1 & 1d & 5 & 16 & 82 & $181.8^{\circ}$ & $77.34 \%$ \\
\hline 2 & 1d & 10 & 12 & 83 & $182.1^{\circ}$ & $77.80 \%$ \\
\hline 3 & $1 e$ & 5 & 16 & 83 & $184.7^{\circ}$ & $78.00 \%$ \\
\hline 4 & 1e & 10 & 10 & 85 & $184.8^{\circ}$ & $79.35 \%$ \\
\hline 5 & $2 \mathrm{~b}$ & 5 & 15 & 90 & $192.2^{\circ}$ & $80.13 \%$ \\
\hline 6 & $2 \mathrm{~b}$ & 10 & 9 & 90 & $192.3^{\circ}$ & $80.59 \%$ \\
\hline 7 & $2 c$ & 5 & 13 & 91 & $193.0^{\circ}$ & $83.21 \%$ \\
\hline 8 & $2 c$ & 10 & 8 & 92 & $193.2^{\circ}$ & $84.19 \%$ \\
\hline 9 & $3 e$ & 5 & 18 & 77 & $174.3^{\circ}$ & $71.57 \%$ \\
\hline 10 & $3 e$ & 10 & 14 & 79 & $174.6^{\circ}$ & $72.45 \%$ \\
\hline 11 & $3 f$ & 5 & 17 & 80 & $176.8^{\circ}$ & $73.62 \%$ \\
\hline 12 & $3 f$ & 10 & 12 & 80 & $177.1^{\circ}$ & $73.69 \%$ \\
\hline
\end{tabular}

${ }^{\mathrm{a}}$ Isolated yield after purification. $.^{\mathrm{b}} \mathrm{SOR}-\left(c 0.3\right.$ in Acetone at $\left.24^{\circ} \mathrm{C}\right) .{ }^{12 \mathrm{c}}$ Enantiomeric excess (\% ee) for absolute configuration $(2 S 3 R)$ was determined by HPLC analysis using a Phenomenex Chiralpack column by comparison with the HPLC retention time using known standard. ${ }^{13}$

acid derivatives (3e and $\mathbf{3 d}$ ) has OR group at $\beta$ position to the quaternary nitrogen which can help to form hydrogen bonding or attractive van der Waals interaction to get advantage to enhance the enantioselectivity. These novel chiral phase transfer catalysts have high melting points more than $238^{\circ} \mathrm{C}$.

To check the effectiveness of these novel PTCs, these catalysts has been applied for the enantioselective epoxidation of $\alpha, \beta$ - unsaturated chalcone (Figure 4) and for Darzen condensation (Figure 5), in which enantioselective epoxide (4) was synthesized. In both the cases, it has been found that all the novel PTCs resulted in good enantioselectivity. The reason for getting the good enantioselectivity in both the cases may be due to the interaction of oxidant with $\beta$ carbon of substrate (chalcone) from the upside direction in the 1,4 addition to produce $\mathrm{S}$ and R configuration (Figure 6).

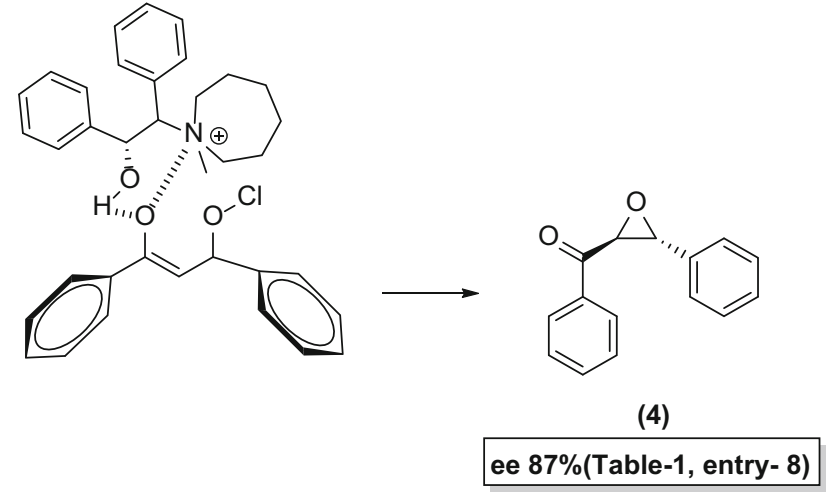

Figure 6. Plausible mechanism for the enantioselective epoxidation by using Novel PTC (2C).

However, the results with mandelic acid derivatives (ee 81-87\%) were markedly better compared to proline 
and tartaric acid derivatives (ee 72-80\%). The proline derivatives (ee 77-80\%) produced better results than the tartaric acid derivatives (ee 72-76\%) (Table 1 and Table 2). In addition to this, in both the applications, among the same category of catalysts, the catalysts with larger cyclic rings $(\mathbf{1 e}, \mathbf{2 c}, \mathbf{3 f})$ proved to give better results than the smaller cyclic rings (1d. $\mathbf{2 b}, \mathbf{3 e})$. The reason may be attributed to the bulkiness or steric hindrance generated on quaternary nitrogen by the larger cyclic groups.

Moreover, the mandelic acid and proline derivatives (1d, 1e, 2b and 2c) have better results (ee 77-87\%) as compared to the tartaric acid derivatives (ee 72-76\%) as these catalysts are not only ion paired with the oxidant ion but also form hydrogen bonding with the help of free $-\mathrm{OH}$ group present at $\beta$ position with respect to quaternary nitrogen (Figure 6). Whereas in tartaric acid derivatives ( $\mathbf{3 e}$ and $\mathbf{3 f}$ ) the hydrogen bonding is not possible because they have the alkoxy group. There was no impact of the quantity of catalysts loaded $(5 \mathrm{~mol} \%$ or $10 \mathrm{~mol} \%$ ) on enantioselectivity and yield except the reaction time (Table 1 and Table 2). The rate of reaction is directly proportional to the quantity of catalyst loaded.

\section{Conclusions}

We have synthesized novel chiral phase transfer catalysts derived from Proline, Mandelic acid and Tartaric acid having cyclic quaternary ammonium salts. We have employed very simple methods (though multistep process) for the synthesis of these chiral phase transfer catalysts by designing the geometry of quaternary Nitrogen. The PTCs have been isolated with desired quality and competitive yield. The structure of PTCs have been confirmed by analytical techniques.

These catalysts are effectual towards the synthesis of enantioselective epoxidation from chalcones and by Darzen condensation. Further applications of these catalysts for asymmetric synthesis are in progress.

\section{Supplementary Information (SI)}

${ }^{1} \mathrm{H}$ and ${ }^{13} \mathrm{C}$ NMR spectra for all the phase transfer catalysts and chiral epoxides (Figures S1 to S60) are available in Supplementary Information. Supplementary Information is available at www.ias.ac.in/chemsci.

\section{Acknowledgements}

The authors are thankful to the M/S Lupin Ltd. and the authorities of Manipal Academy of Higher Education for the research program. We also acknowledge the valuable guidance, support and suggestions from Dr. P. R. Upadhaya and Dr. Vijaya Desai.

\section{References}

1. Freedman H H 1986 Industrial applications of phase transfer catalysis (PTC): past, present and future Pure Appl. Chem. $\mathbf{5 8} 857$

2. (a) Starks C, Liotta C and Halpern M 1994 In Book Review Phase-transfer Catalysis: Fundamentals, Applications and Industrial Perspectives (New York: Chapman \& Hall) Ch. 12; (b) Starks C M 1971 Phase-transfer catalysis. I. Heterogeneous reactions involving anion transfer by quaternary ammonium and phosphonium salts J. Am. Chem. Soc. 93 195; (c) Starks C and Owens R 1973 Phase-transfer catalysis. II. Kinetic details of cyanide displacement on 1-halooctanes J. Am. Chem. Soc. 953613

3. (a) Stinson S C 1992 In wake of new FDA guidelines, most drug firmsare developing single enantiomers, spawning a "chirotechnology" industry Chem. Eng. News 70 46; (b) Metzger J O 1998 Solvent-free organic synthesis Angew. Chem. Int. Ed. 372975

4. (a) Palvolgyi A, Rapi Z, Ozohanics O, Toth G, Keglevich $G$ and Bako P 2018 Synthesis of alkyl $\alpha$ - and $\beta$-D-glucopyranoside-based chiral crown ethers and their application as enantioselective phase-transfer catalysts Res. Chem. Intermed. 44 1627; (b) Wengang G, Xianghui L, Yan L and Can L 2018 Chiral catalysis at the water/oil interface ACS Catal. 8 328; (c) Nemcsok T, Rapi Z, Keglevich G, Grun A and Bako P 2018 Synthesis of D-mannitol-based crown ethers and their application as catalyst in asymmetric phase transfer reactions Chirality 30 407; (d) Woo H M, Young L J, Doyoung K, Geumwoo L, Kyun L J, Suckchang H and Hyeung-Geun P 2018 Enantioselective synthesis of chiral $\alpha$-thio-quaternary stereogenic centers via phasetransfer-catalyzed $\alpha$-alkylationof $\alpha$-acylthiomalonates $J$. Org. Chem. 83 1011; (e) Seunga W, Yong-Gyun K, Baegeun L, Oh Jiin O, Yeonji L, Hyeri G and Keepyung N 2018 Dimeric cinchona ammonium salts with benzophenone linkers: enantioselective phase transfer catalysts for the synthesis of $\alpha$-amino acids $R S C A d v . \mathbf{8}$ 2157

5. (a) Makosza M 2000 Phase-transfer catalysis A general green methodology in organic synthesis Pure Appl. Chem. 72 1399; (b) Dolling U H, Davis P, Grabowski E J J 1984 Efficient catalytic asymmetric alkylations. 1. Enantioselective synthesis of(+)-indacrinone via chiral phase-transfer catalysis J. Am. Chem. Soc. 106 446; (c) Hughes D L, Dolling U-H, Ryan K M, Schoenewaldt E F and Grabowski E J J 1987 Efficient catalytica symmetric alkylations-3. A kinetic and mechanistic study of the enantioselective phase-transfer methylation of 6,7-dichloro-5-methoxy-2-phenyl-1-indanone J. Org. Chem. 52 4745; (d) O'Donnell M J, Benett W D and Shengde W 1989 The stereo selective synthesis of alphaamino acids by phase-transfer catalysis $J$. Am. Chem. Soc. 111 2353; (e) Lipkowitz K B, Cavanaugh M W, Baker B and O'Donnell M J 1991 Theoretical studies in molecular recognition: asymmetric induction of 
benzophenone imine ester enolates by the benzylcinchoninium ion J. Org. Chem. 56 5181; (f) O'Donnell M J, Wu S and Huffman J C 1994 Anew active catalyst species for enantioselective alkylation by phase-transfer catalysis Tetrahedron 50 4507; (g) O'Donnell M J, Shengde W, Irena E and Aiqiao M 1996 Catalytic enantioselective synthesis of alpha-amino acid derivatives byphase-transfer catalysis U.S. Patent 5554753; (h) O'Donnell M J, Esikova I A, Mi A, Shullenberger D F and Wu S 1997 In Phase-Transfer Catalysis 3rd edn. M E Halpern (Ed.) ACS Symposium Series 659; American Chemical Society, Washington, DC, Ch. 10; (i) Corey E J and Noe M C 1996 Kinetic investigations provide additional evidence that an enzyme-like binding pocket is crucial for high enantioselectivity in the bis-cinchona alkaloid catalyzed asymmetric dihydroxylation of olefins J. Am. Chem. Soc. 118 319; (j) Corey E J and Noe M C 1993 Rigid and highly enantioselective catalyst for the dihydroxylation of olefins using osmium tetra oxide clarifies the origin of enantio-specificity $J$. Am. Chem. Soc. 115 12579; (k) Corey E J, Noe M C and Sarshar S 1994 X-ray crystallographic studies provide additional evidence that an enzyme-like binding pocket is crucial to the enantioselective dihydroxylation of olefins by $\mathrm{OsO}_{4}$-bis-cinchona alkaloid complexes Tetrahedron Lett. 35 2861; (1) Corey E J and Noe M C 1996 Improved enantioselective dihydroxylation of bishomoallylic alcohol derivatives using a mechanistically inspired bis-cinchona alkaloid catalyst Tetrahedron Lett. 371735

6. (a) Vachon J and Lacour L 2006 Recent developments in enantioselective phase transfer catalysis using chiral ammonium salts Chim. Int. J. Chem. 60 266; (b) Young K D, Jae C Y, Yoon P H, Ung J C, Oh K K, Yang M J and Kang-Yeoun J 2003 Enantioselective epoxidation of $\alpha$, $\beta$-unsaturated ketones by phase-transfer catalysis using chiral quaternary ammonium salts Synth. Commun. 33 435; (c) Lygo B 2001 In Phase-Transfer Reactions in Rodd's Chemistry of Carbon Compounds Vol. V:
Asymmetric Catalysis M Sainsbury (Ed.) (Oxford: Elsevier Science Ltd.) p. 101; (d) O’Donnell M J 2000 In Asymmetric Phase-Transfer Reactions in Catalytic Asymmetric Synthesis 2nd edn. I Ojima (Ed.) (New York: Verlag Chemie) p. 725; (e) T Shioiri 1997 In Chiral Phase-Transfer Catalysts: A Handbook of PhaseTransfer Catalysis Y Sasson and R Neumann (London: Blackie) p. 462; (f) Helder R, Hummelen J C, Laane R W P M, Wiering J S and Wynberg H 1976 Catalytic asymmetric induction in oxidation reactions-synthesis of optically active epoxides Tetrahedron Lett. 171831

7. Beak P, Kerrick S T, Wu S and Chu J 1994 Complex induced proximity effects: enantioselective syntheses based on asymmetric deprotonations of N-bocpyrrolidines J. Am. Chem. Soc. 1163231

8. Pauly R, Sasaki N A and Pierre P 1994 A versatile method for the synthesis of (S)- or (R)cycloalkylglycines, (S)- or(R)-N-heterocyclic and $\alpha$, $\beta$-unsaturated $\mathrm{N}$-heterocyclic $\alpha$-amino acids Tetrahedron Lett. 35237

9. Chen K, Navendu J and Driver T G $2013 \mathrm{Rh}_{2}$ (II)catalyzed selective aminomethylene migration from styryl azides Org. Lett. 15824

10. Bilke J L, Moore S P, O’Brien P and Gilday J 2009 Catalytic asymmetric synthesis of piperidines from pyrrolidine: concise synthesis of L-733,060 Org. Lett. 111935

11. Servet T, Bilsen T and Demir A S 2015 Hetero functional magnetic metal-chelate-epoxy supports for the purification and covalent immobilization of benzoyl formate decarboxylase from pseudomonas putida and its carboligation reactivity Chirality 27635

12. Chao Z, Dan Y, Bei Z and Yingming Y 2015 Highly enantioselective epoxidation of $\alpha, \beta$-unsaturated ketones catalyzed by rare-earth amides $\left[\left(\mathrm{Me}_{3} \mathrm{Si}\right)_{2} \mathrm{~N}\right]_{3} \mathrm{RE}(\mu-1)$ $\mathrm{Li}(\mathrm{THF})_{3}$ with phenoxy-functionalized chiral prolinols Org. Lett. 172242

13. Arai S, Tsuge H and Shioiri T 1998 Asymmetric epoxidation of $\alpha, \beta$-unsaturated ketones under phase-transfer catalyzed conditions Tetrahedron Lett. 397563 